\title{
REVISÃO SISTEMÁTICA DA LITERATURA SOBRE A MODELAGEM FUNCIONAL DE PRODUTOS MECATRÔNICOS
}

Thyago Alberto Ullmann (thyago.ullmann@gmail.com) - Programa de Pós-Graduação em Engenharia e Ciências Mecânicas, Universidade Federal de Santa Catarina.

Régis Kovacs Scalice (regis.scalice@ufsc.br) - Programa de Pós-Graduação em Engenharia e Ciências Mecânicas, Universidade Federal de Santa Catarina.

\section{RESUMO}

O desenvolvimento de projetos de produtos mecatrônicos apresenta complexidade ao considerar as fronteiras do domínio existentes entre as disciplinas de mecânica, eletrônica e software. Ao analisar as práticas existentes para o desenvolvimento de projetos para estes produtos, é verificada uma deficiência dos procedimentos atuais em entregar o resultado de forma mais simples e concisa para algumas atividades de projeto, tal qual a modelagem funcional. Na busca pela evolução no processo conceitual de desenvolvimento de produtos mecatrônicos, este artigo tem como direcionamento a identificação do estado da arte para modelagem funcional de produtos mecatrônicos tendo como base a publicação de artigos em periódicos. A busca e a análise dos artigos têm como base os princípios da Revisão Sistemática da Literatura (RSL), possibilitando a identificação, seleção e avaliação crítica de artigos publicados com base no tópico de interesse. A questão de pesquisa a qual este trabalho se orienta é: quais os métodos e ferramentas são utilizados para elaborar a modelagem funcional de um produto mecatrônico? Ao considerar os trabalhos estudados para compor a pesquisa, é verificada a ausência na literatura de um modelo funcional de produtos mecatrônicos, que apresente em um único diagrama as correlações entre as funções pertencentes às diferentes disciplinas envolvidas.

Palavras chave: projeto conceitual, modelagem funcional, produtos mecatrônicos

Área: Ferramentas e métodos de desenvolvimento de produtos e serviços

\section{INTRODUÇÃO}

A modelagem funcional permite descrever os produtos em um nível abstrato, onde é possível visualizar a estrutura do produto por meio das funcionalidades. Analisar o produto em um plano abstrato possibilita uma abrangência na obtenção das soluções. A abstração também pode ser empregada na identificação de restrições fictícias, que possam restringir a utilização de novas tecnologias no produto, materiais e processos de fabricação. Como vantagem, o resultado da análise destes fatores possibilita um melhor entendimento da tarefa de projeto e identificação das funções do produto contribuindo significativamente para o Projeto Conceitual. (ROZENFELD et al., 2006)

Back et al. (2008) se referem a modelagem funcional com o termo síntese funcional e atribuem a origem na década de 1970 citando trabalhos de pesquisadores alemães como: Koller (1985), Pahl e Beitz (1996), Rodenacker (1991) e Roth (1982). Para Ferreira (1997) e Back et al. (2008) a modelagem funcional é representada inicialmente pela função global ou total do produto, sendo esta um resumo do que se deve esperar do produto e normalmente é obtida com a análise dos requisitos funcionais contidos na lista de especificações meta do produto. Rozenfeld et al. (2006) completa que a função global é representada em geral por 
uma transformação com entradas e saídas definidas. BACK et al. (2008) ainda considera que juntamente com a função global são analisadas e definidas as interfaces do sistema, com destaque para interações com outros sistemas técnicos, interações com o usuário e interações com o meio ambiente.

De acordo com Rozenfeld et al. (2006) as entradas e saídas do sistema são classificadas em energia, material e sinal. O sinal é considerado como sendo a forma física na qual uma informação é transportada. O material representa propriedades de forma, massa, cor, condições, entre outros. Já a energia é a responsável pelo transporte ou transformação de matéria e sinal, pois para que o produto realize uma transformação é necessário a existência de um fluxo de energia entrando e saindo do sistema. A estrutura funcional é obtida com o desdobramento da função global em funções menos complexas. Esta estrutura é desdobrada em um processo iterativo até a obtenção de uma representação que atenda o nível de detalhamento requerido.

A correlação do tema modelagem funcional com produtos mecatrônicos pode apresentar conflitos considerando uma questão central neste tipo de produto, onde as decisões de projeto não podem ser tomadas analisando um único domínio, considerando impacto em outros domínios. O desafio está na identificação de uma abordagem que considere a interação dos domínios mecânicos, eletrônicos e de controle. (ALVAREZ CABRERA et al., 2010)

De acordo com Liu et al. (2015) na indústria moderna, produtos complexos e variados são impulsionados pelo avanço da tecnologia e pela diversidade das necessidades dos consumidores. E, para garantir as funções variadas e alcançar um aumento no desempenho demandada pelos consumidores, a integração de produtos com muitas funções ou tecnologias são desenvolvidos para substituir produtos específicos. A integração de produtos específicos em um só artefato tornou-se uma forma comum de inovação.

A integração de produtos específicos pode ser direcionada para análise de produtos mecatrônicos onde atrás do projeto do conceito está a capacidade de transferir domínios complexos da mecânica para eletrônica e para o software, com o objetivo de alcançar uma alta performance e reduzir custos, conforme descrito por Walters, Bradley e Dorey (2000).

Abid et al. (2015) reforçam que o desenvolvimento de sistemas mecatrônicos é complexo em vários níveis. Por um lado, as interações entre diferentes domínios criam dependência funcional e estrutural que geralmente não são gerenciadas pelas ferramentas disponíveis. Em complemento o desenvolvimento de sistemas mecatrônicos envolve a colaboração entre diferentes áreas que exigem abordagens metodológicas que possam reunir as melhores práticas.

A abordagem da modelagem funcional em produtos mecatrônicos direciona para uma flexibilidade na utilização dos conceitos, e Fotso, Wasgin e Rettberg (2012) reforçam que este tema é convencionado para uso na Engenharia Mecânica onde se verificam produtos menos complexos em comparação aos mecatrônicos, principalmente pela ausência de software de controle.

Considerando a importância da modelagem funcional em projetos de produto, e dada ampla aplicabilidade em produtos mecatrônicos, as seções seguintes realizam uma abordagem de trabalhos com direcionamento para o tema em questão alinhado com as propriedades de uma Revisão Sistemática da Literatura. O presente trabalho é divido em quatro seções, onde considera-se a primeira como introdução, a seção dois traz um detalhamento da metodologia utilizada juntamente com informações sobre pesquisa, na seção três é realizada uma análise das práticas identificadas, e ao final, na seção quatro são expostas as considerações finais e projeções de trabalhos futuros. 


\section{METODOLOGIA}

A busca e a análise dos artigos teve como base os princípios da Revisão Sistemática da Literatura (RSL) considerando a pesquisa de Kitchenham et al. (2010) uma das responsáveis pela adaptação da RSL, originalmente da Medicina, para Computação, possibilitando a identificação, seleção e avaliação crítica de artigos publicados com foco em um tópico de interesse. Inicialmente, é definida a questão de pesquisa, e a partir desta, são definidas as palavras-chave e os operadores lógicos. Seguidos estes passos, é possível iniciar a coleta de dados e sua posterior análise.

A questão de pesquisa a qual este trabalho se orienta é: quais os métodos e ferramentas são utilizados para elaborar a modelagem funcional de um produto mecatrônico?

As etapas seguintes da RSL são apresentadas na Figura 1, onde é possível visualizar o fluxo das informações até a obtenção dos artigos que contribuem para a questão da pesquisa.

A etapa 1 descrita na Figura 1 é direcionada com a utilização da base de dados Scopus considerando a sua abrangência e facilidade de uso. A definição dos logaritmos de busca está restrita para título, resumo e palavras chave, e teve como objetivo a verificação de trabalhos que relacionam o a modelagem funcional com produtos mecatrônicos. Foram utilizados como termos de busca as palavras: function model, function modeling, functional modeling, functional model, systems modeling, product architecture, functional basis, functional analysis, functional design, feature-based modeling, product representation, function-based design, functional synthesis, product modeling e systems model sempre acrescidos do termo mechatronic.

A pesquisa foi realizada entre 1 e 30 de agosto de 2016 e direcionada para artigos publicados em periódicos, sendo desconsiderados neste momento trabalhos de congressos denominados na base Scopus como "Conference Paper", e outros artigos citados nos trabalhos identificados.

A etapa de busca resultou em 99 artigos sendo que após a realização do primeiro filtro foram selecionados 86 artigos. Este filtro inicial considera a análise do título, resumo e palavras chave. O segundo filtro dos artigos inclui a leitura da introdução e conclusão, o que resultou em 25 artigos que possuem direcionamento para a questão da pesquisa.

Figura 1 - Processamento RSL. Fonte: Adaptado de Conforto, Amaral e Silva (2011)

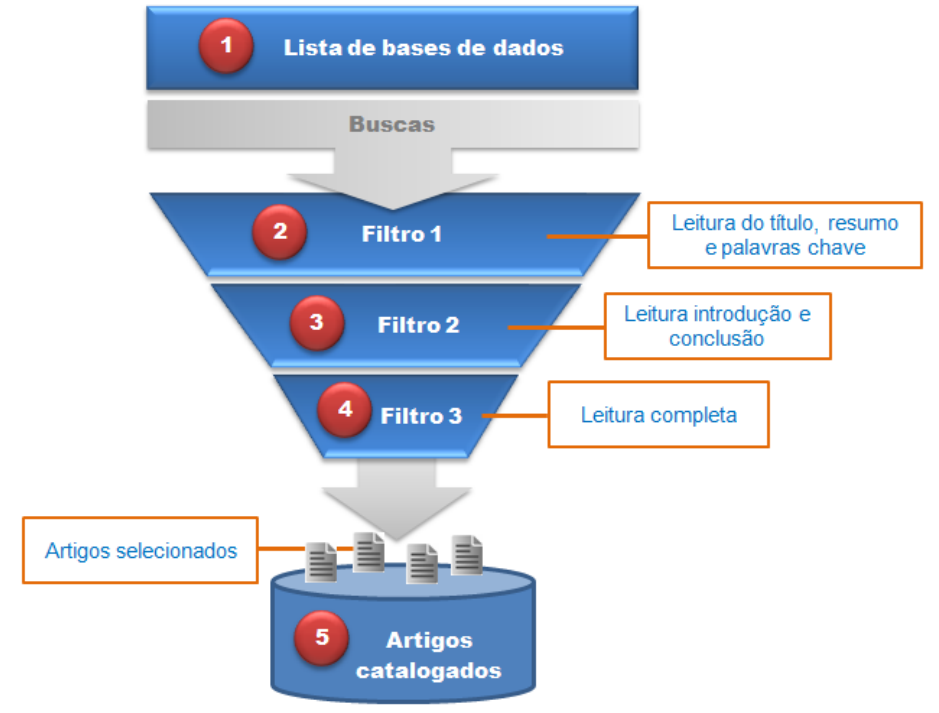


Como critério de inclusão dos artigos há um direcionamento para os trabalhos que desenvolvem práticas que auxiliam a fase inicial do desenvolvimento do projeto de produtos mecatrônicos com foco na análise das funções dos produtos. Com relação a exclusão, para fins de filtragem, foram desconsiderados artigos com direcionamento para um modelo de desenvolvimento de projeto para produtos mecatrônicos, artigos que trabalham com enfoque na comunicação entre as áreas e equipes de projetos, artigos com práticas de desenvolvimento de produtos mecatrônicos para a confiabilidade ou redução de falhas, artigos com práticas para a validação de projeto conceitual e artigos com foco na modelagem específica do software de controle envolvido nos produtos mecatrônicos. Tais artigos divergem da questão da pesquisa.

A terceira etapa do filtro tem como objetivo realizar uma análise detalhada do conteúdo de cada artigo. Nesta última etapa os artigos foram pontuados em uma escala de 1 a 10 indicando a sua correlação com a questão da pesquisa, sendo a escala apresentada no Quadro 1.

O Quadro A1 incluído no apêndice apresenta a relação de artigos classificados após a realização dos filtros com base no título, resumo, palavras chave, introdução e conclusão. Também é indicada a classificação dos artigos após a leitura completa dos conteúdos.

Com a classificação, dois artigos receberam a nota 1 , nove artigos receberam a nota 3 , sete artigos receberam a nota 5, três artigos receberam a nota 7 e quatro artigos receberam a nota 10 .

\section{ANÁLISE DAS PRÁTICAS}

Wu, Leu e Liu (2010) e Leu, Wu e Liu (2009) propõem uma estrutura de decomposição funcional orientada a objetos (Object-Oriented - OO) direcionada para o co-design (design simultâneo) e co-análise (análise simultânea) das funções do produto, estruturas e as relações entre os componentes. A estrutura proposta utiliza uma árvore de funções (function tree) para apresentar o modelo funcional do produto adicionando o uso do HOOM e o FOMM.

Os autores buscam oferecer uma ferramenta que auxilie na comunicação em uma equipe multidisciplinar de projeto através do detalhamento e análise nível a nível do produto considerando partes mecânicas, eletrônicas e software, além de ser uma ponte entre projetistas e engenheiros no desenvolvimento das especificações de projeto.

Quadro 1 - Análise das Pontuações. Fonte: Elaborado pelo Autor, 2017

\begin{tabular}{|c|l|}
\hline NOTA & \multicolumn{1}{c|}{ DESCRIÇÃO DA PONTUAÇÃO } \\
\hline 1 & $\begin{array}{l}\text { Artigos sem abordagem de uma prática específica de modelagem funcional de produtos mecatrônicos } \\
\text { ou que possuem uma abordagem com modelo genérico de desenvolvimento de produtos }\end{array}$ \\
\hline 3 & $\begin{array}{l}\text { Artigos que citam práticas para a modelagem funcional de produtos mecatrônicos mas não possuem } \\
\text { uma explicação específica da ferramenta }\end{array}$ \\
\hline 5 & $\begin{array}{l}\text { Artigos que correlacionam práticas de modelagem funcional de produtos mecatrônicos mas apenas os } \\
\text { vinculam a um modelo integrado de desenvolvimento de produtos }\end{array}$ \\
\hline 7 & $\begin{array}{l}\text { Artigos que correlacionam práticas de modelagem funcional de produtos mecatrônicos e vinculam a } \\
\text { um modelo integrado de desenvolvimento de produtos, porém com pouco detalhamento }\end{array}$ \\
\hline 10 & Artigos que citam e detalham práticas de modelagem funcional de produtos mecatrônicos \\
\hline
\end{tabular}

O hierarchical $\mathrm{OO}$ functional modeling technique é iniciado com a definição da função principal do produto. Após a definição, a subfunção é decomposta em subfunções e cada subfunção deve ser decomposta até a identificação de uma função primitiva. Estas funções 
primitivas são então relacionadas com os objetos. O HOOM é então utilizado para decomposição da estrutura de objetos de alta ordem para objetos primitivos. Os autores exemplificam um objeto primitivo como sendo a placa mãe enquanto um objeto de alta ordem seria um computador.

O FOOM é então utilizado para como técnica de análise e verificação para descrever o mapeamento das relações e implementação de parâmetros entre funções e objetos. A análise dos parâmetros de implementação, refere-se aos fluxos de informação necessários para que os objetos implementem as funções. Existindo três tipos de fluxos: material, energia e sinal.

Os autores complementam o trabalho com um exemplo de aplicação da ferramenta no desenvolvimento de um robô para desarmar bombas.

LIU et al. (2015) apresentam uma metodologia para o projeto conceitual de produtos complexos baseada na análise de modos. Considera-se modo o estado de configuração do sistema comutável para um propósito específico. Como exemplo os autores citam uma lâmpada solar onde ocorre a conversão e armazenamento de energia no modo diurno e conversão de energia em iluminação no modo noturno. Os autores apresentam uma relação entre modo, função e tecnologia através dos três eixos de um sistema cartesiano e abordam a apropriação destes três aspectos em produtos simples que evoluíram com a apropriação de outras tarefas. Como exemplificação é apresentado a modelagem de um relógio esportivo com medição de batimentos cardíacos.

Como sequência ao método de projeto sistemático apresentado por Pahl et al. (2007), os autores apresentam dois métodos para realizar o detalhamento das funções e sub-funções e produtos com vários modos. O primeiro denominado Merging Method (Método de Fusão) é composto por quatro etapas que promovem a síntese entre as funções através dos modos.

O segundo denominado Clustering Method (Método de Agrupamento), também é composto por 4 etapas sendo que o objetivo é estabelecer modos com base na abundância de recursos do sistema.

O trabalho desenvolvido por van Beek, Erden e Tomiyama (2010) relaciona a modelagem funcional com o projeto modular de produtos mecatrônicos. Analisando o desenvolvimento do modelo funcional, os autores utilizam o function-behavior-state (FBS; Função de Comportamento de Estado) como sendo uma abordagem de modelamento baseada nas descrições funcionais e comportamentais de elementos estruturais. A abordagem possui suporte de software através do FBS Modeler. A ferramenta citada permite ao projetista desenvolver o FBS sugerindo decomposições funcionais, associação de funções de nível mais baixo com estruturas físicas e verificar a consistência do modelo. Qualquer função em conflito com as estruturas físicas pode ser detectada e visualizada pelo usuário. O FBS Modeler é composto por dois tipos de conhecimento. O primeiro está direcionado para as características físicas (processos, entidades e relações espaciais das entidades) correspondentes ao comportamento objetivo do sistema. O segundo tipo de conhecimento é direcionado ao nível subjetivo das funcionalidades. Estes conhecimentos são armazenados em duas formas, considerando a como as funções são decompostas em sub-funções e quais as características físicas das funções. Ao projetar um produto com o FBS Modeler, o projetista define e decompõe primeiro as funções necessárias. Então analisa os recursos físicos para realizar as funções. O metodologia desenvolvida pelos autores relaciona o FBS com a design structure matrix (DSM; Matriz de Estrutura do Projeto). Esta matriz apresenta um direcionamento para a modularização do produto no projeto conceitual.

A proposta desenvolvida por Alvarez Cabrera et al. (2010) apresenta uma alternativa à abordagem de integração das metodologias existentes para apoiar o desenvolvimento de 
sistemas mecatrônicos e adiciona uma orientação para o controle de software. Os autores sugerem o uso do FBS (function-behavior-state) para iniciar a modelagem e abrangem a integração com a utilização de outras metodologias como IDEF0, QFD e Modelo V. Estas metodologias traduzem um modelo de informação funcional de alto nível com objetivo fornecer uma visão geral e classificar informações detalhadas de projeto, capturando funções, requisitos e a arquitetura do sistema.

Além das informações de alto nível, os autores apresentam a necessidade de um modelo do sistema de informações de análise como simulações do controlador. Neste sentido é proposto a utilização de técnicas orientadas a objetos como o Systems Modeling Language (SysML) integrado com ferramentas como CATIA e Matlab/Simulink, e a combinação do SysML com o Composable Object (COB; Objeto Composto ). Estas técnicas permitem a construção de modelos rápidos e intuitivos, uma vez que os elementos de modelagem foram desenvolvidos. Considerando o nível arquitetônico, várias visões sobre o sistema geral podem ser visualizados. Essas visões podem ser usadas para capturar e preocupações e exigências das partes interessadas do projeto, favorecendo o projeto simultâneo e reduzindo problemas de comunicação entre a equipe de projeto.

Zheng et al. (2016) apresentam um modelo de interface multidisciplinar em produtos mecatrônicos. O método é composto por três conceitos: classificação da interface, modelagem da interface e definição de regras de compatibilidade das interfaces. De acordo com os autores o modelo de interface multidisciplinar permite uma representação comum para as interfaces definidas pela equipe de projeto membros de especialidades diversas. Assim o modelo proposto garante a consistência da interface e auxilia os projetistas a garantir a correta integração de componentes diferentes. O estudo é iniciado com a análise dos tipos de transferências que ocorrem através de uma interface sendo relacionados: geometria da interface, energia da interface, controle da interface e comunicação de interface. Na sequência são analisadas as configurações das interfaces considerando então três elementos em sistemas mecatrônicos (componente, ambiente e interface). O estudo correlaciona os três elementos de forma que envolve a interface entre componentes, interface entre componentes e o ambiente, interface entre componentes e interface, interface entre interfaces e interface entre interfaces e ambiente. Os autores apresentam estas correlações através do UML .

Mhenni et al. (2014) trabalham uma correlação para a utilização do SysML na modelagem de sistemas mecatrônicos com o Modelo V, considerando as fases de projeto do sistema e a integração do sistema, sendo que o trabalho apresentado tem foco na primeira fase do modelo. A metodologia proposta, o SysML based, visa auxiliar o projetista na fase de projeto do sistema para ter uma modelagem consistente. A metodologia também tenta orientar o projetista frente à variedade de diagramas do SysML, dando uma seqüência de usos desses diagramas em diferentes estágios de projeto.

O processo de modelação SysML based é composto por duas fases e a metodologia é apresentada com o exemplo de desenvolvimento de um equipamento de GPS. A primeira fase é denominada black-box (caixa preta), e tem a função de fornecer um ponto de vista geral do sistema. No contexto das funções, o sétimo estágio desta fase trabalha com o cenário das funções.

A segunda fase, denominada white-box (caixa branca), é composta pelo detalhamento da arquitetura interna do sistema, e direcionando para as funções do exemplo.

O estágio final da fase white-box busca a apresentação da arquitetura física para o produto. 


\section{CONCLUSÃO}

O projeto de desenvolvimento interdisciplinar que ocorre nos produtos mecatrônicos, exige dos projetistas a utilização de diferentes abordagens de modelagem sendo que esta precisam se unir orientadas pelo propósito do projeto. A questão da diversidade na modelagem de funções dentro e entre diferentes disciplinas se traduz em uma barreira central para a uma colaboração mais harmônica, demandando um maior número de diagramas, ampliando tempo de projeto e criando redundâncias de informações desnecessárias. Tal contexto leva a necessidade de novos esforços de pesquisa para um melhor entendimento da literatura, de forma a compilar as informações em benefício ao desenvolvimento dos projetos.

Os artigos analisados permitem a formação de uma base de dados específica disponibilizando acesso a ferramentas e metodologias recentes e que ainda podem ser combinadas para oferecer um melhor desempenho ao trabalhar com sistemas mecatrônicos. É importante ressaltar que a pesquisa não contempla as citações realizadas nos artigos e também não abrange os trabalhos que citam os artigos analisados, mas possibilita identificar as práticas que estão sendo utilizadas para dar suporte à elaboração do projeto conceitual de produtos mecatrônicos tendo como apoio a análise das funções do produto. A partir dos trabalhos analisados é verificado que o tema da modelagem funcional para produtos mecatrônicos não possui uma estruturação no mesmo nível que ocorre para o campo da mecânica, e verifica-se, porém, a oportunidade de desenvolvimento, em trabalhos futuros, de uma sistematização fundamentada na modelagem funcional que possibilite apresentar de forma unificada as correlações entre as diferentes disciplinas existentes nos produtos mecatrônicos.

\section{REFERÊNCIAS}

ABID, H. et al. SysML approach for the integration of mechatronics system within PLM systems. International Journal of Computer Integrated Manufacturing v. 28, n. 9, p. 972-987, 2015.

ALVAREZ CABRERA, A. A. et al. Towards automation of control software: A review of challenges in mechatronic design. Mechatronics v. 20, n. 8, p. 876-886, 2010.

BACK, N. et al. Projeto integrado de produtos: planejamento, concepção e modelagem. Barueri, SP: Manole, 2008.

CONFORTO, E.; AMARAL, D. C.; SILVA, S. L. D. Roteiro para revisão bibliográfica sistemática: aplicação no desenvolvimento de produtos e gerenciamento de projetos. $8^{\circ}$ Congresso Brasileiro de Gestão de Desenvolviemnto de Produto - CNGDP 2011 v. 8, n. 1998, p. 1-12, 2011.

FERREIRA, M. G. G. Utilização De Modelos Para a Representação De Produtos No Projeto Conceitual. Dissertação (Mestrado em Engenharia Mecânica) - Universidade Federal de Santa Catarina. p. 128, 1997.

FOTSO, A. B.; WASGINT R.; RETTBERG, A. State of the art for mechatronic design concepts. Proceedings of 2012 IEEE/ASME 8th IEEE/ASME International Conference on Mechatronic and Embedded Systems and Applications p. 232-240 , 2012.

KITCHENHAM, B. et al. Systematic literature reviews in software engineering - A tertiary study. Information and Software Technology v. 52, n. 8, p. 792-805, ago. 2010.

LEU, M. C.; WU, J. C.; LIU, X. F. Axiomatic functional and object-oriented product design framework. CIRP Annals - Manufacturing Technology v. 58, n. 1, p. 147-152, 2009.00078506 . 
LIU, C. et al. Conceptual design of multi-modal products. Research in Engineering Design v. 26, n. 3, p. 219-234, 2015.

MHENNI, F. et al. A SysML-based methodology for mechatronic systems architectural design. Advanced Engineering Informatics v. 28, n. 3, p. 218-231 , 2014.

PAHL, G. et al. Engineering design. NULL. [S.1.]: Springer, 2007. 3rd ed. 2007.1447160258.

ROZENFELD, H. et al. Gestão do Processo de Desenvolvimento de Produtos: Uma Refêrencia para a Melhoria do Processo. São Paulo: Saraiva, 2006. 541 p.

VAN BEEK, T. J.; ERDEN, M. S.; TOMIYAMA, T. Modular design of mechatronic systems with function modeling. Mechatronics v. 20, n. 8, p. 850-863, 2010.

WALTERS, R. M.; BRADLEY, D. A.; DOREY, A. P. A conceptual study for a computerbased tool to support electronics design in a mechatronic environment. v. 24, p. 51-61, 2000.

WU, J.-C.; LEU, M. C.; LIU, X. F. A Hierarchical Object-oriented Functional Modeling Framework for Co-Design of Mechatronic Products. Concurrent Engineering v. 17, n. 4, p. 245-256, 2010..

ZHENG, C. et al. Multidisciplinary interface model for design of mechatronic systems. Computers in Industry v. 76, n. C, p. 24-37, 2016.978-1-4799-4833-8. 


\section{APÊNDICES}

Quadro A1 - Artigos. Fonte: Elaborado pelo Autor, 2017

\begin{tabular}{|c|c|c|c|c|c|}
\hline త્ర & ڤึ & \multicolumn{2}{|r|}{ Informações artigo } & \multirow{4}{*}{ 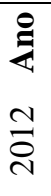 } & 芒 \\
\hline \multirow{3}{*}{3} & \multirow{3}{*}{5} & Autores & Foeken, M.J., Alvarez Cabrera, A.A., Voskuijl, M., Van Tooren, M.J.L. & & \multirow{3}{*}{2} \\
\hline & & Título & Enabling control software generation by using mechatronics modeling primitives & & \\
\hline & & Periódico & Advanced Engineering Informatics & & \\
\hline \multirow{3}{*}{6} & \multirow{3}{*}{10} & Autores & Wu, J.-C., Leu, M.C., Liu, X.F. & \multirow{3}{*}{ 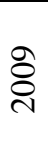 } & \multirow{3}{*}{7} \\
\hline & & Título & $\begin{array}{l}\text { A Hierarchical Object-oriented Functional Modeling Framework for Co-Design } \\
\text { of Mechatronic Products }\end{array}$ & & \\
\hline & & Periódico & Concurrent Engineering Research and Applications & & \\
\hline \multirow{3}{*}{7} & \multirow{3}{*}{10} & Autores & Leu, M.C., Wu, J.C., Liu, X.F. & \multirow{3}{*}{ ஓి } & \multirow{3}{*}{2} \\
\hline & & Título & Axiomatic functional and object-oriented product design framework & & \\
\hline & & Periódico & CIRP Annals - Manufacturing Technology & & \\
\hline \multirow{3}{*}{9} & \multirow{3}{*}{1} & Autores & Buur, J., Myrup Andreasen, M. & \multirow{3}{*}{$\stackrel{\infty}{\mathscr{\infty}}$} & \multirow{3}{*}{49} \\
\hline & & Título & Design models in mechatronic product development & & \\
\hline & & Periódico & Design Studies & & \\
\hline \multirow{3}{*}{12} & \multirow{3}{*}{7} & Autores & Liu, C., Hildre, H.P., Zhang, H., Rølvåg, T. & \multirow{3}{*}{$\frac{n}{8}$} & \multirow{3}{*}{1} \\
\hline & & Título & Conceptual design of multi-modal products & & \\
\hline & & Periódico & Research in Engineering Design & & \\
\hline \multirow{3}{*}{13} & \multirow{3}{*}{5} & Autores & Politze, D.P., Dierssen, S., Wegener, K. & & \\
\hline & & Título & Function module drivers for assessing the similarity between product functions & 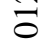 & 1 \\
\hline & & Periódico & CIRP Journal of Manufacturing Science and Technology & & \\
\hline & & Autores & Van Beek, T.J., Erden, M.S., Tomiyama, T. & & \\
\hline 15 & 7 & Título & Modular design of mechatronic systems with function modeling & 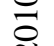 & 42 \\
\hline & & Periódico & Mechatronics & & \\
\hline & & Autores & $\begin{array}{l}\text { Alvarez Cabrera, A.A., Foeken, M.J., Tekin, O.A., (...), Van Houten, F.J.A.M., } \\
\text { Tomiyama, T. }\end{array}$ & & \\
\hline 16 & 7 & Título & $\begin{array}{l}\text { Towards automation of control software: A review of challenges in mechatronic } \\
\text { design }\end{array}$ & 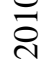 & 29 \\
\hline & & Periódico & Mechatronics & & \\
\hline & & Autores & Walters, R.M., Bradley, D.A., Dorey, A.P. & & \\
\hline 19 & 5 & Título & $\begin{array}{l}\text { Conceptual study for a computer-based tool to support electronics design in a } \\
\text { mechatronic environment }\end{array}$ & 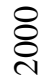 & 0 \\
\hline & & Periódico & Microprocessors and Microsystems & & \\
\hline & & Autores & Zheng, C., Le Duigou, J., Bricogne, M., Eynard, B. & & \\
\hline 36 & 10 & Título & Multidisciplinary interface model for design of mechatronic systems & 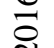 & 0 \\
\hline & & Periódico & Computers in Industry & & \\
\hline & & Autores & Yuan, W., Liu, Y., Zhao, J., Wang, H. & & \\
\hline 40 & 5 & Título & Pattern-based integration of system optimization in mechatronic system design & $\overline{0}$ & 0 \\
\hline & & Periódico & Advances in Engineering Software & & \\
\hline & & Autores & Thramboulidis, K. & & \\
\hline 41 & 3 & Título & $\begin{array}{l}\text { Comments on "A model-based design methodology for the development of } \\
\text { mechatronic systems" }\end{array}$ & $\frac{n}{2}$ & 0 \\
\hline & & Periódico & Mechatronics & & \\
\hline & & Autores & Abid, H., Pernelle, P., Noterman, D., Campagne, J.-P., Ben Amar, C. & & \\
\hline 42 & 5 & Título & SysML approach for the integration of mechatronics system within PLM systems & 0 & 0 \\
\hline & & Periódico & International Journal of Computer Integrated Manufacturing & & \\
\hline & & Autores & Mhenni, F., Choley, J.-Y., Penas, O., Plateaux, R., Hammadi, M. & & \\
\hline 45 & 10 & Título & A SysML-based methodology for mechatronic systems architectural design & $\overline{0}$ & 7 \\
\hline & & Periódico & Advanced Engineering Informatics & & \\
\hline & & Autores & Nomaguchi, Y., Fujita, K. & & \\
\hline 47 & 5 & Título & $\begin{array}{l}\text { Knowledge representation framework for interactive capture and management of } \\
\text { reflection process in product concepts development }\end{array}$ & $\frac{n}{8}$ & 0 \\
\hline & & Periódico & Advanced Engineering Informatics & & \\
\hline
\end{tabular}


Quadro A1 - Artigos (continuação). Fonte: Elaborado pelo Autor, 2017

\begin{tabular}{|c|c|c|c|c|c|}
\hline ฮై & $\stackrel{\frac{\pi}{0}}{\stackrel{2}{Z}}$ & & Informações artigo & $\stackrel{8}{\xi}$ & تُن \\
\hline \multirow{3}{*}{51} & \multirow{3}{*}{3} & Autores & Liu, Y.-S., Fan, H.-R., Jiang, Y.-Q., Cao, Y. & \multirow{3}{*}{$\frac{N}{\stackrel{N}{~}}$} & \multirow{3}{*}{0} \\
\hline & & Título & M-design: system modeling platform for multi-domain complex mechatronics & & \\
\hline & & Periódico & Ruan Jian Xue Bao/Journal of Software & & \\
\hline \multirow{3}{*}{56} & \multirow{3}{*}{3} & Autores & Christophe, F., Sell, R., Coatanéa, E. & \multirow{3}{*}{$\stackrel{\infty}{\stackrel{্}{ٍ ~}}$} & \multirow{3}{*}{10} \\
\hline & & Título & $\begin{array}{l}\text { Conceptual design framework supported by dimensional analysis and system } \\
\text { modelling language }\end{array}$ & & \\
\hline & & Periódico & Estonian Journal of Engineering & & \\
\hline \multirow{3}{*}{62} & \multirow{3}{*}{3} & Autores & Osman, K., Stamenković, D., Lazarević, M. & \multirow{3}{*}{$\stackrel{m}{\frac{m}{2}}$} & \multirow{3}{*}{0} \\
\hline & & Título & $\begin{array}{l}\text { Integration of system design and production processes in robust mechatronic } \\
\text { product architectures development - Extended M-FBFP framework }\end{array}$ & & \\
\hline & & Periódico & Hemijska Industrija & & \\
\hline \multirow{3}{*}{79} & \multirow{3}{*}{1} & Autores & Van Der Auweraer, H., Anthonis, J., De Bruyne, S., Leuridan, J. & \multirow{3}{*}{$\stackrel{m}{\stackrel{n}{\sigma}}$} & \multirow{3}{*}{14} \\
\hline & & Título & Virtual engineering at work: The challenges for designing mechatronic products & & \\
\hline & & Periódico & Engineering with Computers & & \\
\hline \multirow{3}{*}{80} & \multirow{3}{*}{3} & Autores & Behbahani, S., De Silva, C.W. & \multirow{3}{*}{$\frac{\text { n}}{\stackrel{N}{\sigma}}$} & \multirow{3}{*}{1} \\
\hline & & Título & $\begin{array}{l}\text { Automated identification of a mechatronic system model using genetic } \\
\text { programming and bond graphs }\end{array}$ & & \\
\hline & & Periódico & $\begin{array}{l}\text { Journal of Dynamic Systems, Measurement and Control, Transactions of the } \\
\text { ASME }\end{array}$ & & \\
\hline \multirow{3}{*}{81} & \multirow{3}{*}{5} & Autores & Salem, F.A. & \multirow{3}{*}{$\frac{m}{\delta}$} & \multirow{3}{*}{1} \\
\hline & & Título & Mechatronics design of small electric vehicles; research and education & & \\
\hline & & Periódico & International Journal of Mechanical and Mechatronics Engineering & & \\
\hline \multirow{3}{*}{82} & \multirow{3}{*}{3} & Autores & Roy, D. & \multirow{3}{*}{$\stackrel{m}{\stackrel{n}{\sigma}}$} & \multirow{3}{*}{0} \\
\hline & & Título & $\begin{array}{l}\text { Design, system model and development of customized Electronic Light Barriers } \\
\text { for robotic and mechatronic applications }\end{array}$ & & \\
\hline & & Periódico & Robotics and Computer-Integrated Manufacturing & & \\
\hline \multirow{3}{*}{83} & \multirow{3}{*}{3} & Autores & Low, C.Y., Aziz, N., Aldemir, M., (...), Anacker, H., Mellado, M. & \multirow{3}{*}{$\frac{m}{\stackrel{n}{\sigma}}$} & \\
\hline & & Título & Strategy planning for collaborative humanoid soccer robots based on principle & & 1 \\
\hline & & Periódico & Production Engineering & & \\
\hline & & Autores & Nattermann, R.S., Anderl, R. & & \\
\hline 86 & 3 & Título & $\begin{array}{l}\text { Simulation data management approach for developing Adaptronic Systems - The } \\
\text { W-model methodology }\end{array}$ & $\overline{\bar{\delta}}$ & 3 \\
\hline & & Periódico & World Academy of Science, Engineering and Technology & & \\
\hline & & Autores & Vyatkin, V., Hanisch, H.-M., Pang, C., Yang, C.-H. & & \\
\hline 93 & 3 & Título & Closed-loop modeling in future automation system engineering and validation & ஓి & 58 \\
\hline & & Periódico & $\begin{array}{l}\text { IEEE Transactions on Systems, Man and Cybernetics Part C: Applications and } \\
\text { Reviews }\end{array}$ & ণ & \\
\hline
\end{tabular}

